\title{
Comment peut-on être européen ?
}

L'orientalisme spéculaire des Lettres persanes

\section{Céline Spector}

\section{(2) OpenEdition}

\section{Journals}

Édition électronique

URL : http://journals.openedition.org/essais/2027

DOI : 10.4000 /essais. 2027

ISSN : 2276-0970

Éditeur

École doctorale Montaigne Humanités

\section{Édition imprimée}

Date de publication : 15 octobre 2013

Pagination : 71-83

ISBN : 978-2-9544269-1-4

ISSN : 2417-4211

Référence électronique

Céline Spector, «Comment peut-on être européen ? », Essais [En ligne], Hors-série 1 | 2013, mis en ligne le 05 octobre 2020, consulté le 18 octobre 2020. URL : http://journals.openedition.org/essais/ 2027 ; DOI : https://doi.org/10.4000/essais.2027 


\section{Comment peut-on être européen? L'orientalisme spéculaire des Lettres persanes}

\section{Céline Spector}

"Mais que les Lumières soient mortes, voilà qui n’est pas sûr $»^{1}$.

Le parcours philosophique et philologique de Carlo Ginzburg sur l'estrangement témoigne d'une curieuse absence : celle des Lettres persanes et de son philosophe persan. Certes, Carlo Ginzburg n'ignore en rien le chef d'œuvre satirique de Montesquieu. À propos des Lettres philosophiques de Voltaire et d'une référence implicite aux Voyages de Gulliver, il rappelle que Swift lui-même s'inspire de l'histoire des trois anneaux exposée par Fontenelle : "Ainsi presque tout est imitation. L'idée des Lettres persanes est prise de celle de l'Espion Turc $»^{2}$. Dans un autre article, Carlo Ginzburg mentionne les Athenian letters, où Cléander n'est pas une invention originale. On peut toujours suivre le jeu de pistes, remonter d'origine en origine ou de source en source : "Aujourd'hui, les Athenian letters font tout de suite penser aux Lettres persanes. Mais le modèle des Athenian letters, [...] n'était pas le livre de Montesquieu mais celui dont Montesquieu s'était inspiré : l'esploratore turco de Gian Paolo Marana (1681), que les traductions et imitations en français et en anglais avaient diffusé dans toute l'Europe ». Dans les deux cas, Lettres persanes et Espion turc, l'artifice narratif est le même : « Le regard corrosif de Montesquieu, ici et là annoncé par le libertin Marana (par exemple dans la description de l'Eucharistie) se porte sans les comprendre sur les habitudes sociales qui nous entourent, en dévoilant par ce truchement leur absurdité,

1 Carlo Ginzburg, "Tolérance et commerce. Auerbach lit Voltaire ", in Le Fil et les traces. Vrai faux fictif, trad. Martin Rueff, Paris, Verdier, 2006, p. 190.

2 Carlo Ginzburg, "L'estrangement. Préhistoire d'un procédé littéraire ", À distance. Neuf essais sur le point de vue en histoire, trad. P.-A. Fabre, Paris, Gallimard, 2001, p. 15-36. Voir le compterendu de Perry Anderson in la London Review of Books (vol. 34, n 8, 26 avril 2012), qui relève cet étrange silence. 
leur arbitraire $»^{3}$. Ici comme ailleurs, l'étranger (l'espion, le voyageur) s'enquiert des usages afin de "défamiliariser un présent que nous avons tendance à considérer comme allant de soi ", ou de "nous familiariser avec un passé dont la physionomie quotidienne nous échappe $"^{4}$.

Comment démystifier le champ politique, social, ou religieux, comment déjouer les faux-semblants et dépasser l'écorce des apparences ? Pour Carlo Ginzburg, l'estrangement est « susceptible de constituer un antidote efficace à un risque qui nous guette tous : celui de tenir la réalité (nous compris) pour sûre " ${ }^{5}$. Dans un texte sur Voltaire lu par Auerbach, les conséquences antipositivistes du dispositif apparaissent au grand jour ${ }^{6}$. Dans les Lettres philosophiques notamment, Voltaire a fait en sorte que les gestes les plus évidents deviennent étranges, opaques, absurdes, "comme s'ils étaient vus par les yeux d'un étranger, d'un sauvage ou d'un philosophe ignorant $"$.

Le philosophe ignorant: ne nous aide-t-il pas à y voir plus clair par sa cécité même, par son ignorance ? Dans cet esprit, la présente étude tentera une exploration, fondée sur une question née du dispositif spéculaire convoqué par Montesquieu (Orient/Occident, Asie/Europe, Ispahan/Paris). Cette question sera le miroir de la question posée à Rica dénudé, mis à nu, selon l'expression privilégiée par Carlo Ginzburg, volontairement dépouillé de ses habits persans : non plus " comment peut-on être Persan ? ", qui n'est pas la véritable question posée par Montesquieu, mais plutôt " comment peut-on être européen ? ", question plus que jamais troublante. Trois figures de l'estrangement seront distinguées ici : 1. l'estrangement comme stratégie de défamiliarisation ; 2. l'estrangement comme dispositif spéculaire ; 3. l'estrangement comme figure de l'impossible retour à soi ou de l'impossible lucidité sur soi, comme révélateur de l'angle mort de la philosophie et de la culture.

\section{L'estrangement comme stratégie de défamiliarisation}

Parues sans nom d'auteur en 1721 à Amsterdam, les Lettres persanes sont introduites au moyen d'un procédé diaphane énoncé dans la préface : Montesquieu s'y fait passer pour l'éditeur-traducteur de documents communiqués par des voyageurs persans, auxquels se seraient ajoutés quelques secrets, prétendument dérobés à leur insu. Beaucoup a été dit sur le dispositif épistolaire qui permet à l'auteur anonyme des Lettres persanes de mettre en scène l'étrangeté des mœurs européennes en usant d'un double regard persan - regard du grand

3 Carlo Ginzburg, "Anarachasis interroge les indigènes ", in Le Fil et les traces, op. cit., p. 223.

4 Ibid., p. 224.

5 Carlo Ginzburg, «L'estrangement. Préhistoire d'un procédé littéraire », art. cit., p. 36.

6 Carlo Ginzburg, "Tolérance et commerce. Auerbach lit Voltaire ", in Le Fil et les traces, op. cit., p. 175-176.

7 Ibid., p. 177. 
seigneur, philosophe curieux mais désabusé (Usbek), regard enthousiaste et plus naif du jeune novice qui l'accompagne (Rica). Beaucoup d'encre mais peu d'attention, au fond, à ce dédoublement des regards et des voix, qui crée d'emblée une double figure de l'inquiétante étrangete ${ }^{8}$. Usbek a fui la persécution après avoir combattu la corruption à la Cour; c'est un tyran domestique, aveugle sur les contradictions de son propre désir. Rica, lui, est dans la force de la jeunesse, prêt à prendre un nouveau " pli " et à oublier sa patrie - figure de l'ingratitude qui est aussi une figure de l'émancipation'. Deux Persans s'exilent donc pour s'enrichir, les premiers, des lumières de l'Occident. Face à la nouveauté des institutions qui se dévoilent à leurs yeux, les voyageurs décodent, décryptent, décèlent, dévoilent. Usbek en cisèle la formule à la lettre 48 : «tout m’intéresse, tout m'étonne : je suis comme un enfant dont les organes encore tendres sont vivement frappés par les moindres objets $»^{10}$.

Que voient donc les voyageurs persans ? Ou plutôt comment voient-ils, selon quels biais culturels et religieux dévoilent-ils l'Occident qui se livre à eux précisément sans voile (littéralement : le fait que les femmes y soient " en liberté » est le premier impact du choc des civilisations) ? Ce qu'ils voient est, soi-disant, la vérité mise à nu, la vérité sans fard dans le jeu mondain du " doux commerce » ou d'une " communication » prétendument transparente

8 Voir Suzanne Gearhart, The Open Boundary of History and Fiction. A critical approach to the French Enlightenment, Princeton, Princeton University Press, 1984, chap. 3. Lauteur interprète la posture contradictoire d'Usbek comme celle de l'outsider qui tente en vain d'accéder au savoir universel en restant prisonnier de sa culture (d'insider), là où Rica a perçu, comme Barthes, Lévi-Strauss ou Lacan, que la culture était un empire de signes. Selon S. Gearhart, Montesquieu révèle que sa posture d'outsider est intenable : son contact avec une culture étrangère accroît la conscience malheureuse de sa limitation (p. 96-97).

9 Nous fournirons la numérotation usuelle des Lettres persanes (désormais $L P$ ), établie à partir de l'édition de 1758 (Lettres persanes, Paul Vernière (éd.) mise à jour par Catherine Volpilhac-Auger, Paris, Librairie générale française, "Le livre de poche ", 2005) en complétant entre parenthèses par la numérotation (et la pagination) effectuée sur l'édition de 1721, in Euvres complètes de Montesquieu, t. I, sous la direction de Catherine Volpilhac-Auger et Philip Stewart, Oxford, Voltaire Foundation, 2004, LP, 27 (25), p. 202 (orthographe et ponctuation modernisées).

$10 L P, 48$ (46), p. 253. Usbek et Rica se disent bien reçus en Occident, ce qui permet de camper le dispositif épistolaire de l'estrangement, car les outsiders pourront devenir insiders à leur façon : " notre air étranger n'offense plus personne, nous jouissons même de la surprise où l'on est, de nous trouver quelque politesse : car les Français n'imaginent pas que notre climat produise des hommes ; cependant, il faut l'avouer, ils valent bien la peine qu’on les détrompe ". La Préface évoque un possible retournement : "Les Persans qui écrivent ici, étaient logés avec moi ; nous passions notre vie ensemble. Comme ils me regardaient comme un homme d'un autre monde, ils ne me cachaient rien. En effet des gens transplantés de si loin, ne pouvaient plus avoir de secrets : ils me communiquaient la plupart de leurs lettres : je les copiai ; J'en surpris même quelques-uns, dont ils se seraient bien gardés de me faire confidence ; tant elles étaient mortifiantes pour la vanité, et la jalousie persane. Je ne fais donc que l'office de traducteur : toute ma peine a été de mettre l'ouvrage à nos mœurs : j'ai soulagé le lecteur du langage asiatique autant que je l'ai pu ; et l'ai sauvé d'une infinité d'expressions sublimes, qui l'auraient ennuyé jusques dans les nues" (p. 138). 
entre les corps et les esprits (doux commerce opposé en miroir à la gravité sans sociabilité et à l'opacité sans joie du despotisme asiatique $\left.{ }^{11}\right)$. Serait-ce donc cela, Paris ? Ce monde où les hommes et les femmes se "livrent " et se " communiquent ", c'est-à-dire sont polis, civils, galants, sociables ? Un lieu de plaisirs et de liberté où hommes et femmes seraient prémunis du carcan des rites, des pesanteurs religieuses et des servitudes politiques?

Un instant suffit pour nous en dissuader. Dès la première lettre parisienne, la messe est dite : Paris n'est nullement un lieu de sincérité et de liberté, délivré de vains salamalecs et de formules "sublimes", aussi insipides que fleuries. Paris est l'empire des signes, d'autres codes, d'autres rites. On y simule et on y dissimule avec autant d'ardeur qu'en Perse, quoique sur un ton plus enjoué. Invoquant cette transparence, la préface nous livrait en ce sens une fausse piste. Que révèle donc l'estrangement - le regard persan(t) ? À l'évidence, dans le registre de la satire, les ridicules, les travers, les vices, les faux-semblants de ce que les Persans nomment le " noir Occident ». La phénoménologie sociale fait son œuvre : le regard des Persans sélectionne ce qui frappe, étonne, amuse, indigne; et le premier phénomène, véritable épiphanie, est celui des rapports sociaux eux-mêmes, livrés d'emblée au théâtre et à l'Opéra, dans la forme de la pantomime qui ne fait qu' exagérer l'artifice et l'hypocrisie, la simulation et la dissimulation, mais aussi les illusions et les prestidigitations qui caractérisent les rapports sociaux en Occident ${ }^{12}$. L'estrangement dévoile les ressorts de la comédie humaine, le théâtre du monde dont les signes sont habituellement interprétés à la lumière d'un code plus ou moins inconscient (incorporé socialement et culturellement). L'estrangement est donc un procédé de dé-codage, qui permet d'encoder autrement.

On pourrait multiplier les exemples. C'est Rica qui ouvre le bal à la lettre 24 (à Ibben) : "Ne crois pas que je puisse, quant à présent, te parler à fond des mœurs et des coutumes européennes : je n'en ai moi-même qu'une légère idée, et je n'ai eu à peine que le temps de m'étonner ». Ce temps de l'étonnement n’est pas encore écoulé que déjà les signes les plus visibles doivent être réinterprétés. Les rapports politiques et religieux seraient-ils duplices?

«Le roi de France est le plus puissant prince de l'Europe. [...]

D'ailleurs ce roi est un grand magicien; il exerce son empire sur l'esprit même de ses sujets ; il les fait penser comme il veut. S'il n'a qu'un million d'écus dans son trésor, et qu'il en ait besoin de deux, il n'a qu'à leur persuader qu'un écu en vaut deux, et ils le croient. [...]

11 La préface en témoigne : «Il y a une chose qui m’a souvent étonné, rapporte l'éditeur-traducteurnarrateur ; c'est de voir ces Persans quelquefois aussi instruits que moi-même, des mœurs, et des manières de la nation, jusqu'à en connaître les plus fines circonstances ; et à remarquer des choses, qui, je suis sûr, ont échappé à bien des Allemands, qui ont voyagé en France. J'attribue cela au long séjour qu'ils y ont fait. D'autant qu'il est plus facile à un Asiatique de s'instruire des mœurs des Français dans un an qu'il ne l'est à un Français de s'instruire des mœurs des Asiatiques dans quatre ; parce que les uns se livrent autant que les autres se communiquent peu » (p. 139).

12 Je me permets de renvoyer à Céline Spector, Montesquieu. Les «Lettres persanes », Paris, PUF, 1997. 
Ce que je te dis de ce prince ne doit pas t'étonner : il y a un autre magicien, plus fort que lui, qui n'est pas moins maître de son esprit qu'il l'est lui-même de celui des autres. Ce magicien s'appelle le Pape. Tantôt il lui fait croire que trois ne sont qu'un, que le pain qu'on mange n'est pas du pain, ou que le vin qu'on boit n'est pas du vin, et mille autres choses de cette espèce ${ }^{13}$.

Tout joue ici sur la figure du retournement : l'éclaircissement dû aux "lumières européennes " semble rencontrer l'obscurité ou la pénombre - la superstition de l'Europe elle-même, qui n'est pas seulement religieuse mais aussi politique et civile ${ }^{14}$. Comment peut-on être Européen, et qu'est-ce qu'être Européen au fond ? Ce que dévoile l'estrangement est l'intrusion de la magie, de la duperie, de l'intolérance et de la servitude au beau milieu de ce qui fait la puissance et le renom de l'Europe policée et son fleuron (Paris!) - sa civilisation, sa politique, son économie florissante, ses salons, ses femmes, ses académies savantes, ses sciences et ses arts. Les Lettres persanes ne sont pas seulement un manifeste des Lumières, le premier peut-être, qui témoignerait de la grandeur du "siècle de Louis XIV »; c'est un manifeste de ce que ce siècle de Louis XIV a encore d'ignorant, de faux, de duplice, de barbare. La portée subversive de l'estrangement est perceptible dans la chute des fausses idoles : "Le pape est le chef des Chrétiens. C'est une vieille idole qu'on encense par habitude... $»^{15}$. L'Europe chrétienne n'est pas une terre de paix : "aussi puis-je t'assurer qu'il n'y a jamais eu de royaume où il y ait eu tant de guerres civiles que dans celui du Christ ${ }^{16}$. L'Europe n'est pas non plus une terre de tolérance, et l'on sera sensible à la tonalité voltairienne du propos : « il y a certains dervis qui n'entendent point raillerie, et qui font brûler un homme comme de la paille. Quand on tombe entre les mains de ces gens-là, heureux celui qui a toujours prié Dieu avec de petites grains de bois à la main, qui a porté sur lui deux morceaux de draps attachés à deux rubans (allusion au scapulaire, morceaux d'étoffe attachés par des rubans sur la poitrine, signes de dévotion à la Vierge), et qui a été quelquefois dans une province qu'on appelle la Galice [allusion au pèlerinage de Saint Jacques de Compostelle] ! Sans cela un pauvre diable est bien embarrassé [...] Il serait en cendres avant que l'on eût seulement pensé à l'écouter ${ }^{17}$.

13 LP, 24 (22), p. 191-193. La lettre est inspirée de Marana, L'Espion turc, op. cit., t. V, lettre 5 : Du pouvoir qu'ont les rois en France de guérir les écrouelles ; et t. I, lettre 12 : «ils mangent d'un certain pain [...] où ils s'imaginent que leur Messie est réellement présent [...] As-tu jamais rien vu de si fou?".

14 Voir LP, 31 (29), de Rhédi : "Je m’instruis des secrets du commerce, des intérêts des princes, de la forme de leur gouvernement ; je ne néglige pas même les superstitions européennes : je m’applique à la médecine, à la physique, à l'astronomie ; j'étudie les arts ; enfin je sors des nuages qui couvraient mes yeux dans le pays de ma naissance » (p. 215).

$15 L P, 29(27)$, p. 208.

16 Ibid., p. 209.

17 Ibid., p. 210. 
Lieu d'apparition de la vérité des sciences (vérité galiléenne, vérité cartésienne, qu'une lettre d'Usbek magnifie ${ }^{18}$ ), l'Europe est aussi la terre brûlée de l'Inquisition : " Ils font dans leur sentence un petit compliment à ceux qui sont revêtus d'une chemise de souffre, et leur disent qu'ils sont bien fâchés de les voir si mal habillés, qu'ils sont doux, qu'ils abhorrent le sang et sont au désespoir de les avoir condamnés. Mais, pour se consoler, ils confisquent tous les biens de ces malheureux à leur profit ». Le retournement est complet, puisque la lettre 29 se conclut en ces termes : "Heureuse la terre qui est habitée par les enfants des prophètes! Ces tristes spectacles nous sont inconnus. La sainte religion que les anges y ont apportée se défend par sa vérité même : elle n'a point besoin de ces moyens violents pour se maintenir ${ }^{19}$.

Mais le véritable moment qui permet d'assigner à l'estrangement son rôle opératoire intervient dans la célèbre lettre qui met en lumière l'artifice littéraire et le procédé ethnologique du dépouillement. À la lettre 30, Rica ôte son habit persan et quitte ses couleurs bariolées pour un plus sobre vêtement à l'européenne. Il se trouve alors plongé, dit-il, dans un " néant affreux » - celui de l'invisibilité sociale, sur la scène fébrile de la lutte pour la reconnaissance ${ }^{20}$. L'expérience de pensée du dépouillement de l'écorce (l'habit bariolé) vaudrait révélation de l'essence des rapports sociaux en Occident : la vanité y règne en maîtresse d'illusion et sert de ressort au despotisme, qui établit son empire sur les esprits et sur les cœurs ${ }^{21}$. Ce qui se joue en Occident n'est autre que ce que Rousseau identifiera quelques années plus tard, et l'on pourrait déjà en dire, en un sens, ce qu'Axel Honneth écrira à propos de Rousseau : Montesquieu a perçu le ressort d'une critique de la civilisation centrée sur le phénomène de l'aliénation ${ }^{22}$.

La mise en abyme est surprenante : l'estrangement comme aliénation révèle le phénomène d'aliénation qui constitue les rapports sociaux corrompus en Europe. La critique des fourvoiements de la modernité s'amorce dans les Lettres persanes avant de se déployer dans le Discours sur les sciences et les arts et le Discours sur l'origine et les fondements de l'inégalité parmi les hommes. L'estrangement rend manifeste un fait social total : l'essor de la civilisation rend l'homme dépendant de convoitises artificielles et de désirs contradictoires. La civilisation

18 LP, 97 (94).

19 Ibid., p. 211-212.

20 LP, 30 (28), p. 214.

21 "Le roi de France est le plus puissant prince de l'Europe. Il n'a point de mines d'or comme le roi d'Espagne, son voisin ; mais il a plus de richesses que lui, parce qu'il les tire de la vanité de ses sujets, plus inépuisable que ses mines. On lui a vu entreprendre et soutenir de grandes guerres, n'ayant d'autres fonds que des tiers d'honneur à vendre, et, par un prodige de l'orgueil humain, ses troupes se trouvaient payées, ses places, munies, et ses flottes, équipées " ( $L P$, 24/22, p. 191).

22 Axel Honneth, "Les pathologies du social. Tradition et actualité de la philosophie sociale " (1994), in La Société du mépris. Vers une nouvelle Théorie critique, trad. O. Voirol, P. Rusch et A. Dupeyrix, Paris, La Découverte, 2006, p. 39-100. 
asservit l'homme et le rive au règne des apparences, au moment même où l'essor des sciences et les arts, du luxe, des modes, de la politesse et du goût, voile sa servitude et l'accentue encore en la rendant non seulement supportable mais plaisante. L'estrangement révèle donc l'essence des rapports sociaux aliénés en Occident et livre une véritable philosophie sociale avant la lettre : s'aliéner revient ici à perdre son identité, son rapport non corrompu à l'altérité, à se perdre dans les besoins factices et les méandres inauthentiques d'une course effrénée à la reconnaissance, qui est aussi une course aveugle à la servitude ${ }^{23}$.

Face à cette perversion de la société civile dont les figures emblématiques sont au gré du roman la coquette, le nouvelliste, l'Académicien, le casuiste, le libertin, le petit-maître ou le bel esprit, Montesquieu ne livre certes pas comme Rousseau la toile de fond (l'état de nature) qui permet de percevoir, par contraste, les pathologies de la civilisation. Mais il revient à la procédure de l'estrangement de jouer en quelque sorte le rôle d'analogon de l'état de nature : le regard persan, mais plus encore l'absence de regard sur le persan dépouillé de son habit persan, font apparaître la corruption, la mutilation ou l'aliénation constitutives de la modernité en Occident ${ }^{24}$. L'invisibilité sociale de Rica, désormais sans atours, le révèle : dans la capitale du luxe et du goût, du règne des femmes arbitres des réputations, où les stratégies de distinction doivent opérer dans l'immédiateté et l'anonymat de la rencontre, la dynamique sociale s'emballe dans les méandres de l'inauthenticité et de la quête infinie du prestige. Mus par l'amourpropre, les individus sont conduits à simuler (les talents, les attraits, les signes de distinction) qui leur procureront, espèrent-ils, un surcroît de reconnaissance, sans jamais apaiser ce désir infini et sans trêve, et ce jusqu'à la mort.

Bien entendu, cette mise à nu ne s'opère au bénéfice de personne - et surtout pas de l'Orient, polygame, superstitieux et despotique. L'anticléricalisme virulent de Montesquieu ne donne certes pas à voir la pureté d'un Islam préservé de la corruption, de la mutilation et de l'aliénation européennes. La différence Orient/Occident est aussi celle qui sépare mutilation réelle et mutilation symbolique. La chute de la Lettre 57 d'Usbek à Rhédi en témoigne. Il existe certes en Occident des " dervis » - prêtres qui ne respectent pas leurs vœux, ou des casuistes qui s'enrichissent en métamorphosant, tels des alchimistes, les péchés mortels en péchés véniels. Mais est-ce si grave ? Usbek livre le dernier

23 Voir notamment Stéphane Haber, L'Aliénation. Vie sociale et expérience de la dépossession, Paris, PUF, 2007 ; L'Homme dépossédé. Une tradition critique, de Marx à Honneth, Paris, CNRS. Éditions, 2009. Et en ce qui concerne Rousseau, Bronisław Baczko, « Rousseau et l'aliénation sociale ", Annales de la Société Jean-Jacques Rousseau, vol. XXXV, 1959-1962, p. 223-237 ; Barbara Carnevali, "La faute à l'amour-propre. Aliénation et authenticité chez Rousseau ", Annales de la Société Jean-Jacques Rousseau, t. XLVIII, 2008, p. 79-103.

24 Voir Florian Nicodème, "Corruption", "mutilation", "pathologie" : réflexions sur le statut de Rousseau dans la Théorie critique (Horkheimer, Adorno, Honneth) ", in Céline Spector (éd.), Modernités de Rousseau, Lumières, $\mathrm{n}^{\circ} 15,1^{\mathrm{er}}$ semestre 2010. 
mot de l'histoire : "Mon père, dis-je, cela est fort bon ; mais comment vous accommodez-vous avec le Ciel ? Si le Sophia avait à sa cour un homme qui fit à son égard ce que vous faites contre votre Dieu, qui mît de la différence entre ses ordres, et qui apprît à ses sujets dans quel cas ils doivent les exécuter, et dans quel autre ils peuvent les violer, il le ferait empaler sur l'heure $»^{25}$.

Crudité de la violence nue, cruauté des imams et des maîtres de sérail, vérité brutale de la mutilation réelle (et non symbolique) du " despotisme oriental $»^{26}$ : dans les Lettres persanes, Montesquieu nous dévoile aussi le régime de la domination pure, qui ne souffre ni différé ni différence, le régime de l'empalement et de l'émasculation, le régime des eunuques. Les stratégies retorses des Occidentaux paraissent alors un peu moins brutales : ruses et simulacres, faux-semblants et dissimulations, toutes ces pathologies de la civilisation aliènent la liberté sans l'anéantir. L'estrangement ne renvoie donc pas au relativisme, encore moins au nihilisme. Il invite plutôt à un dépassement pascalien - retournement du "vain " au "sain ", logique de la raison des effets ou du bel ordre de la concupiscence.

Aussi faut-il en venir au second sens de l'estrangement. La question du miroir se pose ici : jusqu’où joue la structure spéculaire entre Orient et Occident?

\section{L'estrangement-miroir}

À l'évidence, l'estrangement joue comme un miroir, plus précisément comme un miroir que nous tiendrions face à nous ; le dispositif épistolaire polyphonique nous révèle ce qui était dans l'angle mort. Or ce jeu de miroirs livre d'abord une étrange symétrie, car venus du despotisme oriental, les Persans voient ce à quoi les Français risquent de demeurer aveugles : les signes du despotisme à la fin du règne de Louis XIV, voire encore sous la Régence, à la suite de la banqueroute de Law (cet Écossais venu redresser les finances de la France, dont les années au pouvoir se sont soldées par une spéculation immense). Ce que révèle le jeu de miroirs, c'est la tendance au nivellement de la société française, désormais gouvernée par l'argent $(25,84)$, le pouvoir des nouveaux parvenus, financiers et fermiers généraux $(98,48)$, le déclin des

$25 L P, 57$ (55), p. 282.

26 Sur la construction du concept, voir Robert Koebner, « Despot and Despotism: Vicissitudes of a Political Term ", Journal of the Warburg and Courtauld Institutes, 15, 1951, p. 275-302 ; Franco Venturi, "Oriental Despotism ", Journal of the History of Ideas, 24, 1963, p. 133-142; Melvin Richter, "Despotism ", in Philip P. Wiener (éd.), Dictionary of the History of Ideas, 4 vols, NY, 1973, II, p. 1-18 ; Thomas Kaiser, "The Evil Empire? The debate on Turkish Despotism in Eighteenth-Century French Political Culture ", The Journal of Modern History, vol. $72, \mathrm{n}^{\circ}$ 1, Mar. 2000, p. 6-34. Sur son usage chez Montesquieu, voir notamment Elie Carcassonne, Montesquieu et le problème de la Constitution française au XVIII siècle, Paris, 1927 (Genève, Slatkine reprints, 1970) ; David Young, "Montesquieu’s View of Despotism and His Use of Travel Literature ", Review of Politics, 40, 1978, p. 392-405. 
autorités traditionnelles dont font partie les Parlements $(92,140)$, la noblesse, les pères de famille $(86,129)$. En un mot c'est l'honneur, balayé par le règne de la faveur et de l'argent $(24,88)^{27}$. Une " chaîne secrète " fait ainsi communiquer Paris et Ispahan, le réel occidental et la fiction orientale : Paris n'est pas seulement une fête, c'est aussi un lieu de perdition, une sorte de Babylone, pour la noblesse domestiquée et désargentée notamment.

Mais la structure en miroir révèle d'autres arcanes encore, et il serait tentant de relire les Lettres persanes, sinon comme un roman à clé, du moins comme une mise en scène à décors multiples. Un parallèle existe entre la Chair et le Ciel, le sérail et le couven ${ }^{28}$. Écrivant aux eunuques qui gardent son sérail, Usbek insiste de manière fanatique sur la pureté, la vertu et l'obéissance fidèle à " la Loi ", qui est en réalité sa loi. Les "fidèles » eunuques, noirs ou blancs, accomplissent la "volonté sacrée » d'Usbek; le sérail est un " temple sacré " où les femmes s'éduquent par la "mortification des sens "; Usbek est un mari jaloux qui punit le « sacrilège » de l'adultère. Le sérail, au fond, est donc aussi un couvent. Or est-ce si propre à l'Orient ? En Espagne, apprend-on, on garde tout aussi jalousement les femmes, et la religion de l'honneur sert de prétexte à l'oppression : ce sont cette fois les moines franciscains, les novices ou d'autres chaperons, qui font office d'eunuques ${ }^{29}$.

Ainsi Montesquieu ouvre-t-il une brèche, qui se révèle être une ligne de faille : se pourrait-il que le harem dévoile le sort des fidèles, se pourrait-il que son maître absent soit, à sa façon, un Dieu caché ? On s'autorisera à croire que l'auteur des Lettres persanes a usé de la stratégie de l'estrangement pour mieux vulgariser l'amour divin en amour profane, et qu'il a ainsi subtilement illustré l'insulte faite par le Christianisme à la nature de l'homme, sinon de la femme. Figures mutilées, les eunuques sont des créatures étranges, et l'on entend encore l'écho de la célèbre formule de Paul Valéry, " comment expliquer tous ces eunuques? " Mais dans l'un de ses opuscules de jeunesse, «Les prêtres dans le paganisme ", Montesquieu comparait précisément les moines et les prêtres chrétiens aux eunuques ${ }^{30}$.

L'idéal ascétique serait-il l'effet d'une ruse, celle du désir de domination, le jeu sournois et cruel qui trahit la force des faibles, bref, une morale d'esclave ? La lecture nietzschéenne semble ici pertinente : Montesquieu n’a de cesse de révéler dans la structure du sérail les ressorts de la morale du ressentiment, où ceux qui

27 Cf. Jean Ehrard, "Le despotisme dans les Lettres persanes ", Archives des lettres modernes, n 116, 1970, p. 33-50 ; A. Grosrichard, Structure du Sérail. La fiction du despotisme asiatique dans l'Occident classique, Paris, Seuil, 1979.

28 Diana J. Schaub, Erotic Liberalism. Women and Revolution in Montesquieu's "Persian Letters", Londres, Rowman \& Littlefield Publishers, 1995, chap. 5.

$29 \quad L P, 78(75)$, p. 344-345.

30 Voir $M P, 2004$ ( Quelques fragments d'un ouvrage que j'avais fait sur les prêtres dans le paganisme, que j'ai jeté au feu »). 
sont opprimés oppriment à leur tour et usent sans pitié de la "Vertu » et de la "Loi " comme instruments de leur domination. Ambition servile, morale d'émasculés, donc - celle dont la noblesse risque aussi de devenir l'esclave, du moins si elle se complaît dans le rôle de serviteur du bon plaisir du Roi ${ }^{31}$.

\section{L'estrangement, libération de l'aliénation?}

Nous pourrions nous en tenir là, et en un sens, cela dirait déjà toute la fécondité du dispositif de l'estrangement, la valeur heuristique d'un procédé qui n'est pas seulement littéraire mais aussi philosophique - et l'on rendra hommage à Carlo Ginzburg qui en a si subtilement décrits les ressorts. Mais surgit une autre question, très simple au fond : qui détient la vérité philosophique de l'ouvrage ? Qui peut sortir de l'aliénation?

Usbek n'est pas le vrai philosophe. Le philosophe ignorant désireux de s'éclairer reste aveugle, irréductiblement. C'est la fin tragique du roman, et la révolte du sérail. Les femmes tentent in fine de se délivrer de l'oppression. Mais alors qui est le, la véritable philosophe ? Serait-ce une femme, Roxane?

Ce que la polyphonie rend en effet possible n'est pas seulement la variation des points de vue et la diversité des voix (comme dans le superbe dialogue à propos des avantages et des inconvénients du progrès des arts et des sciences, qui offre une vision prophétique de la dialectique négative de la raison et des Lumières $^{32}$ ). Ce que la polyphonie permet, plus profondément peut-être, est aussi l'apparition d'un décalage entre le discours rationnel de la philosophie et la conduite aveuglée par l'empire des sens. Usbek, le "philosophe ", prononce au fil des lettres toute une série d'énoncés parfaitement rationnels sur la nature du pouvoir ou la nature de l'homme. Mais aveuglé par la jalousie, il manque de la lucidité requise pour appliquer ce discours à sa propre situation de maître de sérail ${ }^{33}$. Ainsi lors d'un discours sur l'ivresse et la consolation : la Loi coranique qui prohibe l'alcool, faite "pour nous rendre plus juste ", écrit Usbek, " ne sert souvent qu'à nous rendre plus coupable ». Ne faut-il pas, suggère-t-il alors, " traiter l'homme comme sensible au lieu de le traiter comme raisonnable ${ }^{34}$ ? Dans le même esprit, l’" Essai sur la dépopulation » déplore que la

31 Cela semble d'autant plus évident quand les lettres finales sur le sérail (147-161) sont replacées dans l'ordre chronologique : la plainte de Roxane est écrite en mars 1720 et aurait été reçue par Usbek quatre ou cinq mois plus tard, à peu près au moment où il reçoit la missive de Rica sur le Parlement (juillet 1720). Cf. MP, 596 : " Je disais : "Le gouvernement despotique gêne les talents des sujets et des grands hommes, comme le pouvoir des hommes gêne les talents des femmes" ".

32 Voir LP, 105-106 (102-103, p. 416-423).

33 Voir Jean-Patrice Courtois, "Comment Roxane devient philosophe. Romanesque de l'illisible et sexuation des concepts dans les Lettres persanes ", La lecture littéraire, $\mathrm{n}^{\circ} 3$, janvier 1999, p. 27-47 ; Jean Goldzink, Montesquieu et les passions, Paris, PUF, 2001, p. 25.

$34 L P, 33(31)$, p. 219. 
polygamie et la servitude des femmes dépeuplent le monde, en raison de la finitude du désir masculin, que la sollicitation répétée jusqu'à satiété mène au dégoût, plutôt qu’à la jouissance ${ }^{35}$.

Telle serait la nouvelle dimension du phénomène : l'estrangement du philosophe à lui-même - autre figure de l'aliénation. Dans l'une des lettres les plus politiques de l'ouvrage, placée en son cœur même (la lettre 80), Usbek énonce les avantages associés à un régime modéré, libre et non despotique. Le meilleur régime est celui dont l'art de gouverner contraint le moins possible les passions. De la part de Montesquieu, la farce est savoureuse : il revient au despote de nous livrer l'éloge de la démocratie (en l'occurrence de la monarchie tempérée, qui préserve la liberté) ! Le grand seigneur, maître de sérail, aurait compris que dans les États despotiques régis par la seule crainte, "le Prince, qui est la Loi même, [est] moins maître que partout ailleurs ". La formule suivante frappe comme une prophétie : dans ces régimes dont la stabilité est factice, «le moindre accident produit une grande révolution ${ }^{36}$.

La contradiction est donc logée au cœur du roman, en la personne du philosophe ignorant, despote faussement émancipé et faussement éclairé des lumières d'Occident. Lui qui croyait pouvoir dire, en quittant ses femmes : "Je vous plains, Roxane. Votre chasteté si longtemps éprouvée, méritait un époux qui ne vous eût jamais quittée, et qui pût lui-même réprimer les désirs que votre seule vertu sait soumettre ${ }^{37}$, le lucide Usbek, se trouve confronté à la trahison de Roxane et à la révolte du sérail. Roxane l'infidèle se rebelle : "Comment as-tu pensé que je fusse assez crédule pour m’imaginer que je ne fusse dans le monde que pour adorer tes caprices? que pendant que tu te permets tout, tu eusses le droit d'affliger mes désirs ? Non! J'ai pu vivre dans la servitude, mais j'ai toujours été libre : j'ai réformé tes lois sur celles de la nature, et mon esprit s'est toujours tenu dans l'indépendance ${ }^{38}$. Cri de la nature contre la Loi religieuse et politique, cri de la Liberté contre l'oppression : Roxane le paye de sa vie, mais son suicide héroïque brise l'itération de l'éternelle aliénation. Roxane est la véritable Philosophe, au sens du moins que les Lumières donneront à ce terme : elle est celle par qui le scandale arrive, celle qui dénonce les hypocrisies, les préjugés et les faux-semblants, celle qui démystifie l'autorité et arrache le masque de l'illusion vertueuse. Loin de fuir les climats que son mari qualifiait "d'empoisonnés » par l'impudeur ${ }^{39}$, la voilà qui s'empoisonne, pour mieux fuir la pudeur que ce mauvais mari impose tyranniquement.

Cette " conclusion " des Lettres persanes est révélatrice : Roxane devient philosophe en révélant l'affinité entre nature, liberté et satisfaction de ses désirs,

\footnotetext{
$35 L P, 117$ (113) et 119 (115) sur les effets symétriques de la continence et de la polygamie.

$36 L P, 80(78)$, p. 353.

37 LP, 26 (24), p. 200-201.

38 LP, $161(150)$, p. 544.

39 LP, 26 (24), p. 198.
} 
contre une définition factice, despotique et phallocratique de la vertu et de l'honneur. Vérité philosophique, mensonge romanesque (d'où vient subitement cette voix, d'une femme qui n'est jamais sorti de son sérail ?) ${ }^{40}$. Telle est l'heuristique de l'estrangement: la "révélation " des mécanismes d'assujettissement nous éclaire et nous libère. Au-delà des fausses certitudes (grandeur de l'Occident, misère de l'Orient), Montesquieu nous livre l'histoire des ambivalences de notre condition, et décèle les risques de servitude qui nous menacent. Il n'y a sans doute pas d'autre promesse sans tragique illusion.

Céline Spector

EA $4574 \mathrm{SPH}$

Université Michel de Montaigne Bordeaux 3

celine.spector@u-bordeaux3.fr

\section{Résumé}

Appliquant aux Lettres persanes de Montesquieu la réflexion de Carlo Ginzburg sur la démystification de la vie ordinaire, cette contribution entend distinguer trois figures de l'estrangement: 1. l'estrangement comme stratégie de défamiliarisation ; 2. l'estrangement comme dispositif spéculaire ; 3. l'estrangement comme figure de l'impossible lucidité sur soi, comme révélateur de l'angle mort de la philosophie et de la culture.

\section{Mots-clés}

Montesquieu, Aliénation, Europe, Orient, Féminisme.

40 Comme l'écrit justement Jean-Patrice Courtois, « la polyphonie autorise le déploiement dans le temps de l'ironie tragique " "Comment Roxane devient philosophe. Romanesque de l'illisible et sexuation des concepts dans les Lettres persanes ", La lecture littéraire, $\mathrm{n}^{\circ} 3$, janvier 1999, p. 27-47, ici p. 32). Comme l'a montré J.-P. Courtois, on peut penser les rapports entre roman et philosophie des passions en se situant à l'instant tragique du dénouement de l'intrigue, qui passe par le devenir-philosophe de l'un des personnages (Roxane). Les possibilités stratégiques infinies de la polyphonie épistolaire produisent une situation méta-épistolaire dans laquelle Roxane donne réponse à toute une série de lettres qu'elle n'a pas reçues et que, sur la scène de la fiction, elle ne pouvait pas recevoir. Roxane paye ainsi de sa vie l'accession au langage philosophique, jusque là inconnu au despote. Comme l'écrit Anne-Emmanuelle Berger, " À voileur, voileuse et demi : Roxane exhibe la structure réversible du voile, dissimule son jeu de celui qui la cache, et retourne l'accessoire de l'interdit en instrument de transgression. Le concept se déchire et le rideau tombe " (Comment peut-on être persane ? ", Contretemps, $\mathrm{n}^{\circ}$ 2-3, été-hiver 1997, p. 76-96, ici p. 89). Certaines lectures féministes se sont nourries de ce retournement tragique. D'autres s'appuient sur la forme du roman : Montesquieu aurait inventé un genre qui peut s'adresser aux femmes; il aurait mis en scène une féminisation de la philosophie. Cf. Dena Goodman, Criticism in Action: Enlightenment Experiments in Political Writing, Ithaca, Cornell University Press, 1989, p. 2. 


\section{Abstract}

Applying to Montesquieu's Persian Letters Carlo Ginzburg's theory (the unveiling of ordinary life), this paper will highlight three concepts of estrangement : 1. estrangement as a strategy of defamiliarization; 2. estrangement as a specular device; 3. estrangement as an impossible path to self-awareness, revealing the blind spot of both philosophy and culture.

\section{Keywords}

Montesquieu, Alienation, Europe, Asia, Feminism. 